\title{
Models of quadratic quantum algebras and their relation to classical superintegrable systems
}

\author{
E. G, Kalnins, ${ }^{1}$ W. Miller, Jr., ${ }^{2}$ and S. Post $^{2}$ \\ ${ }^{1}$ Department of Mathematics, \\ University of Waikato, Hamilton, New Zealand. \\ 2 School of Mathematics, University of Minnesota, \\ Minneapolis, Minnesota, U.S.A.
}

\begin{abstract}
We show how to construct realizations (models) of quadratic algebras for $2 \mathrm{D}$ second order superintegrable systems in terms of differential or difference operators in one variable. We demonstrate how various models of the quantum algebras arise naturally from models of the Poisson algebras for the corresponding classical superintegrable system. These techniques extend to quadratic algebras related to superintegrable systems in $n$ dimensions and are intimately related to multivariable orthogonal polynomials.

PACS numbers: 02.00.00, 02.20.Qs,02.30.Ik, 03.65.Fd
\end{abstract}

\section{INTRODUCTION}

The distinct classical and quantum second order superintegrable systems on real or complex spaces of constant curvature in two dimensions have been classified [1]. (Recall that a second order superintegrable system in $n$ dimensions is one that admits $2 n-1$ functionally independent constants of the motion quadratic in the momentum variables, the maximum possible, [2-4].) Indeed, all 2D second order superintegrable systems on all manifolds are known and the classification for nondegenerate potentials on 3D conformally flat spaces is virtually complete, [5-11]. Characteristic of these systems is that the first and second order constants of the motion generate a finite dimensional algebra, polynomially closed under commutation, the quadratic algebra. In several recent papers $[12,13]$ the authors have launched a study of the irreducible representations of these algebras and their applications via models of the representations, in terms of differential and difference operators. In this paper, the first part of which is expository, we describe this model construction in simple 
cases and show how models for quantum systems follow directly from models for the classical systems. Model construction works for systems in all dimensions, [14], but here we restrict attention to the $2 \mathrm{D}$ case to make the presentation more transparent. The final sections on elliptic models contain new results.

\section{AN EXAMPLE}

We will use the following simple degenerate (1-parameter potential) superintegrable system to motivate this article. Consider the classical Hamiltonian on the two sphere

$$
\mathcal{H}=J_{1}^{2}+J_{2}^{2}+J_{3}^{2}+\frac{\alpha}{z^{2}}
$$

where $J_{1}=y p_{z}-z p_{y}, J_{2}=z p_{x}-x p_{z}, J_{3}=x p_{y}-y p_{x}$, and $x^{2}+y^{2}+z^{2}=1$. If we seek all first and second order constants of the motion for this classical Hamiltonian, we find three possibilities in addition to $\mathcal{H}$ itself viz.

$$
A_{1}=J_{1}^{2}+\frac{\alpha}{2 z^{2}}\left(1+y^{2}-x^{2}\right), \quad A_{2}=J_{1} J_{2}-\frac{\alpha x y}{z^{2}}, \quad X=J_{3}
$$

The set $X^{2}, \mathcal{H}, A_{1}$ and $A_{2}$ is linearly independent, but functionally dependent via

$$
A_{1}\left(\mathcal{H}-A_{1}-X^{2}\right)-A_{2}^{2}-\frac{\alpha}{2}\left(X^{2}+\mathcal{H}\right)+\frac{\alpha^{2}}{4}=0
$$

and satisfies the Poisson algebra relations

$$
\left\{X, A_{1}\right\}=-2 A_{2},\left\{X, A_{2}\right\}=-\mathcal{H}+X^{2}+2 A_{1},\left\{A_{1}, A_{2}\right\}=-X\left(2 A_{1}+\alpha\right) .
$$

The quantum version of this system is uniquely determined from the classical system through simple quantization rules [15]. In this case

$$
H=J_{1}^{2}+J_{2}^{2}+J_{3}^{2}+\frac{\alpha}{z^{2}}
$$

where $J_{1}=y \partial_{z}-z \partial_{y}, J_{2}=z \partial_{x}-x \partial_{z}$ and $J_{3}=x \partial_{y}-y \partial_{x}$. The symmetries of the corresponding Schrödinger equation $H \Psi=E \Psi$ are the analogues of the associated constants of the motion viz

$$
A_{1}=J_{1}^{2}+\frac{\alpha}{2 z^{2}}\left(1+y^{2}-x^{2}\right), A_{2}=\frac{1}{2}\left\{J_{1}, J_{2}\right\}-\frac{\alpha x y}{z^{2}}, X=J_{3} .
$$


The quadratic algebra generated by these symmetries is

$$
\left[X, A_{1}\right]=-2 A_{2},\left[X, A_{2}\right]=X^{2}+2 A_{1}-H,\left[A_{1}, A_{2}\right]=-\left\{A_{1}, X\right\}-\left(\frac{1}{2}+\alpha\right) X .
$$

Here, $\{A, B\}=A B+B A$ is the operator symmetrizer. The analogue of the classical constraint is the Casimir condition

$$
\begin{gathered}
\frac{1}{3}\left(X^{2} A_{1}+X A_{1} X+A_{1} X^{2}\right)+A_{1}^{2}+A_{2}^{2}-\left(\frac{3}{2} \alpha+\frac{11}{12}\right) X^{2} \\
+H\left(-A_{1}+\frac{\alpha}{2}-\frac{1}{6}\right)-\frac{2}{3} A_{1}-\frac{\alpha}{2}\left(\frac{\alpha}{2}+1\right)=0 .
\end{gathered}
$$

What is this algebra good for? If we study the spectral resolutions of the symmetry operators for this physical system, e.g., the energy spectrum, the representation theory of the algebra can be used to compute the spectra algebraically and relate the various bases to each other, [16-22]. Also the representation theory for these algebras yields representations other than those that occur in the physical case of the Schrödinger equation, and these have independent interest. As an example we present a one variable model of algebra $(2),(3)$ :

$$
\begin{gathered}
A_{1}=t(t+1)^{2} \partial_{t}^{2}+\left((2-a-m) t^{2}+2(1-m) t+(a-m)\right) \partial_{t}+m(a-1) t+a(m+1)-m-\frac{3}{8}-\frac{1}{2} a^{2} \\
A_{2}=i t\left(1-t^{2}\right) \partial_{t}^{2}+i\left((a+m-2) t^{2}+a-m\right) \partial_{t}-i m(a-1) t \\
X=i\left(t \partial_{t}-m\right), H=-(m+1-a)^{2}+\frac{1}{4}
\end{gathered}
$$

where $\alpha=\frac{1}{4}-a^{2}$. These operators satisfy identically all the relations for the corresponding quantum algebra. This corresponds to irreducible representations of the quadratic algebra, and since $H$ is in the center of the algebra it is a constant in the model. To determine the spectral resolutions of the various operators, we merely solve the eigenvalue differential equations. Indeed solving $A_{1} \Psi_{n}=\chi_{n} \Psi_{n}$ for the finite dimensional irreducible representations we find

$$
\chi_{n}=\frac{1}{2}\left(\frac{1}{4}-a^{2}\right)-\left(n-a+\frac{1}{2}\right)^{2}, \quad n=0,1, . ., m
$$

with corresponding unnormalized eigenfunctions the Jacobi Polynomials

$$
\Psi_{n}(t)=(1+t)_{2}^{n} F_{1}\left(\begin{array}{ll}
n-a & n-m \\
a-m & ;-t
\end{array}\right) .
$$

The spectral resolution for of $X$ is trivial in the model: $X f_{n}=i(n-m) f_{n}, f_{n}(t)=t^{n}$, $n=0,1, \cdots, m$. From this we see that (6) is a generating function for the expansion of the $A_{1}$-basis in terms of the $X$-basis. 
How can such simple models be be found and classified? To answer the question we return to the classical system with this potential and Hamiltonian $\mathcal{H}$. We look for models of the corresponding Poisson algebra on a two dimensional symplectic manifold, the analog of a one variable quantum model. We choose one of our canonical variables to be $X=c$ and take $\beta$ as the canonical (momentum) variable conjugate to $c$. We require $\mathcal{H}=E$, a constant, and look for a realization $A_{1}(c, \beta), A_{2}(c, \beta)$ such that the the Poisson algebra relations (1) remain unchanged but with respect to the new Poisson bracket

$$
\{F, G\}=-\partial_{c} F \partial_{\beta} G+\partial_{\beta} F \partial_{c} G \text {. }
$$

Relations (1) and this new Poisson bracket lead to differential equations for $A_{1}(c, \beta), A_{2}(c, \beta)$ which are easy to solve. The solution is

$$
\begin{gathered}
A_{1}=\frac{1}{2}\left(E-c^{2}\right)+\frac{1}{2}\left[\left(c^{2}-(E+\alpha)^{2}\right] \sin 2 \beta+2 i \alpha \cos 2 \beta\right], \\
A_{2}=\frac{1}{2}\left[\left(c^{2}-(E+\alpha)^{2}\right] \cos 2 \beta-2 i \sqrt{\alpha} \sin 2 \beta\right], X=c .
\end{gathered}
$$

Under the normal rules of quantization, viz $c \rightarrow c, \beta \rightarrow \partial_{c}$, we might expect that there would be a corresponding one variable quantum model. However this leads naturally to a model in terms of difference operators as we would then expect that

$$
\cos 2 \beta \rightarrow \cos \left(2 \partial_{c}\right), \sin 2 \beta \rightarrow \sin \left(2 \partial_{c}\right),
$$

both of which are difference operators. (Note that $e^{\alpha \partial_{c}}$ can be interpreted as translation by $\alpha$ in the variable $c$, via a Taylor expansion.) However if we use the hodograph transformation of classical mechanics, viz $q \rightarrow p, p \rightarrow-q$ (which preserves the Poisson bracket relations), we could elect to quantize according to

$$
c \rightarrow \partial_{c}, \beta \rightarrow-c
$$

(At the quantum level this operation is essentially the Fourier transform.) Now we get a one variable model in which $A_{1}$ and $A_{2}$ are realized as second order differential operators and $X$ is a first order differential operator. To within a gauge transformation this is just the model (4) with $t=e^{i c}$. (Here the classical model gives us the leading terms in the quantum model and then we make lower order adjustments to satisfy the quantum relations (2), (3).) 
We can extend this approach by looking for additional realizations of the classical Poisson algebra. Indeed with a new choice of variables $A_{1}=s$ a model of the Poisson algebra is

$$
\begin{gathered}
A_{1}=s, A_{2}=\sqrt{\left(s-\frac{\alpha}{2}\right)\left(E-s-\frac{\alpha}{2}\right)} \sin \left(2 \sqrt{s+\frac{\alpha}{2}} \beta\right), \\
X=\sqrt{\frac{\left(s-\frac{\alpha}{2}\right)\left(E-s-\frac{\alpha}{2}\right)}{s+\frac{\alpha}{2}}} \cos \left(2 \sqrt{s+\frac{\alpha}{2}} \beta\right),
\end{gathered}
$$

where $\beta$ is the momentum variable conjugate to $s$. By analogy with our previous case it does not appear to be possible to imply from this representation that there is a quantum realization which can be expressed in terms of differential operators. Instead we have only a realization in terms of difference operators. Indeed we have the model

$$
\begin{aligned}
& A_{1}=\left(t^{2}-\frac{\alpha}{2}\right), X=h(t) T_{i}+m(t) T_{-i} \\
& A_{2}=-\frac{i}{2}(i+2 t) h(t) T_{i}+\frac{i}{2}(-i+2 t) T_{-i}
\end{aligned}
$$

where $T_{\alpha} F(t)=F(t+\alpha)$ and $h(t), m(t)$ are constrained by

$$
h(t) m(t+i)=\frac{1}{4} \frac{\left(\alpha-t^{2}-i t\right)\left(t^{2}+i t-E\right)}{t(t+i)} .
$$

A convenient choice is

$$
h(t)=i \frac{\left(\frac{1}{2}-a-i t\right)\left(\mu+a-\frac{1}{2}-i t\right)}{2 t}, m(t)=-i \frac{\left(\frac{1}{2}-a+i t\right)\left(\mu+a-\frac{1}{2}+i t\right)}{2 t},
$$

where $E=-(\mu-1+a)^{2}+1 / 4$. Now for $\tau=i t$ the spectral decomposition of $X$ is $X p_{n}(\tau)=(\mu+2 n) p_{n}(\tau)$ where

$$
p_{n}(\tau)={ }_{3} F_{2}\left(\begin{array}{lll}
-n & \frac{1}{2}-a-\tau & \frac{1}{2}-a+\tau \\
\mu & 1-a
\end{array} ; 1\right),
$$

a special case of the family of dual Hahn polynomials, [23], page 332 .

We can proceed further with these ideas. Indeed if we take $S=2\left(A_{1}-i A_{2}\right)-\mathcal{H}+X^{2}-\alpha$ and $\mathcal{H}$ as our new classical variables then a suitable classical model is

$$
\begin{gathered}
S=r, X=-2 i(r+\alpha) \beta, \\
A_{1}+i A_{2}=8(r+\alpha)^{2} \beta^{4}+2(r+\alpha)(3 \alpha+r+2) \beta^{2}-\frac{(r+E)(\alpha-E)}{2(r+\alpha)}+\frac{\alpha}{2},
\end{gathered}
$$


where $\beta$ is the momentum variable conjugate to $r$. This suggests that there is a quantum realization in terms of fourth order differential operators via the association $r \rightarrow r, \beta \rightarrow \partial_{r}$. In fact, we have the realization

$$
\begin{gathered}
S=r, X=-2 i(r+\alpha) \partial_{r}+2 i \\
A_{1}+i A_{2}=8(r+\alpha)^{3} \partial_{r}^{4}+2(r+\alpha)(3 \alpha+r+2 E+9) \partial_{r}^{2}-2(r+5 \alpha+4 E+18) \partial_{r} \\
+2+\frac{E}{2}+\frac{E^{2}+2 E(9-\alpha)+(\alpha+12)(\alpha+6)}{2(r+\alpha)} .
\end{gathered}
$$

As we showed in Reference 13, by a simple canonical transformation of the classical model we can induce a transformation of this quantum model into a symmetrical form such that $A_{1}$ and $A_{2}$ are second order differential operators. This becomes formally similar to the model (4), but here only infinite dimensional unitary representations of the quadratic algebra can be constructed.

\section{CONNECTION WITH SEPARATION OF VARIABLES}

What is special about the one parameter models that we have chosen? In terms of separation of variables, the classical and quantum versions of this problem separate in exactly the same coordinate systems [24]. Indeed the classical Hamilton-Jacobi equation separates additively and the quantum Schrödinger eigenvalue equation separates multiplicatively. Further, each separable system is characterized by an associated constant of the motion. In the operator case our models enable us to find the spectral resolutions for those operators that define the various possible multiplicative variable separations in the original quantum mechanical problem. For our example physical system on the complex two sphere the coordinates are given in Reference 24 as:

1. Polar coordinates of type 1 . This corresponds to a choice of coordinates

$$
x=\sin \theta \cos \varphi, y=\sin \theta \sin \varphi, z=\cos \theta
$$

The associated model is (4).

2. Polar coordinates of type 2. This corresponds to a choice of coordinates

$$
x=\cos \rho, y=\sin \rho \cos \psi, z=\sin \rho \sin \psi .
$$

The associated model is (7). 
3. Horospherical coordinates.

$$
x=\frac{i}{2}\left(v+\frac{1}{v}\left(u^{2}-1\right)\right), y=\frac{1}{2}\left(v+\frac{1}{v}\left(u^{2}+1\right)\right), z=i \frac{u}{v} .
$$

The associated model is (9).

4. Degenerate elliptic coordinates.

$$
\begin{gathered}
i x-y=\frac{1}{\cosh a \cosh b}, i x+y=-\frac{1}{\cosh a}-\frac{1}{\cosh b}+\frac{1}{\cosh a \cosh b}, \\
z=\tanh a \tanh b .
\end{gathered}
$$

5. Elliptical coordinates.

$$
x^{2}=\frac{\left(\rho-e_{1}\right)\left(\nu-e_{1}\right)}{\left(e_{1}-e_{2}\right)\left(e_{1}-e_{3}\right)}, y^{2}=\frac{\left(\rho-e_{2}\right)\left(\nu-e_{2}\right)}{\left(e_{2}-e_{1}\right)\left(e_{2}-e_{3}\right)}, z^{2}=\frac{\left(\rho-e_{3}\right)\left(\nu-e_{3}\right)}{\left(e_{3}-e_{2}\right)\left(e_{3}-e_{1}\right)} .
$$

We will consider this case in the next section.

We have already found models for cases [1]-[3], In case [4] the constant of the motion that characterizes the separation of variables is

$$
L=2\left(A_{1}+i A_{2}\right)-\mathcal{H}=\left(J_{1}+i J_{2}\right)^{2}-J_{3}^{2}-\frac{1}{2} \alpha-\frac{\alpha x(x+i y)}{z^{2}} .
$$

This constant together with $A_{1}-i A_{2}, \mathcal{H}$ and $X$ form a basis for the Poisson algebra. If we now look for a classical model for which $L=\ell$, and $\beta$ is the canonical variable conjugate to $\ell$, we can proceed in the usual manner and obtain

$$
L=\ell, X=i \sqrt{\ell} \tanh (2 \sqrt{\ell} \beta), A_{1}-i A_{2}=-\frac{1}{2}(\ell+E)-\alpha+(\ell+E+\alpha) \cosh ^{2}(2 \sqrt{\ell} \beta) .
$$

This suggests that if we take $\ell \rightarrow \ell^{\prime 2}$ and use the quantization identification $2 \sqrt{\ell} \beta \rightarrow \beta^{\prime}$ there is a realization of the corresponding quantum algebra via the correspondence $\beta^{\prime} \rightarrow t, \ell^{\prime} \rightarrow \partial_{t}$. Indeed we obtain

$$
\begin{gathered}
L=\partial_{t}^{2}+\frac{J}{\sinh t \cosh t} \partial_{t}+\frac{J^{2} / 2+E+\alpha}{\sinh ^{2} t}, X=-i(\tanh t) \partial_{t} \\
A_{1}-i A_{2}=\frac{1}{2} \sinh ^{2} t \partial_{t}^{2}+\frac{1}{2}[(1-J) \sinh t \cosh t+J \tanh t] \partial_{t} \\
+\frac{1}{2} E \cosh ^{2} t+\left(\frac{\alpha}{2}+\frac{J^{2}}{4}\right) \sinh ^{2} t .
\end{gathered}
$$

To within a gauge transformation, this is just a reparametrization of model (4) again. 
To make the connection with separation of variables for the corresponding superintegrable system clearer, observe that the Schrödinger equation on the two sphere in degenerate elliptic coordinates is

$$
\left[\partial_{a}^{2}-\partial_{b}^{2}+\alpha\left(\frac{1}{\sinh ^{2} a}-\frac{1}{\sinh ^{2} b}\right)-E\left(\frac{1}{\cosh ^{2} a}-\frac{1}{\cosh ^{2} b}\right)\right] \Psi=0 .
$$

Thus the separation equations are

$$
\left(\partial_{\lambda}^{2}+\frac{\alpha}{\sinh ^{2} \lambda}-\frac{E}{\cosh ^{2} \lambda}-\mu\right) \Lambda(\lambda)=0, \quad \lambda=a, b .
$$

Note that the eigenvalue equation for $L$ in our one variable model is

$$
\left(\partial_{t}^{2}+\frac{J(J+2)}{4 \cosh ^{2} t}+\frac{4(E+\alpha)+J(J+2)}{4 \sinh ^{2} t}-\mu\right) T(t)=0 .
$$

With the identification $E=-J(J+2) / 4$ this is exactly a copy of one separation equation for the superintegrable system. In addition we have seen that the one variable model implied by degenerate elliptic coordinates is essentially the one we already know.

\section{THE ELLIPTICAL MODEL}

In each of the cases [1]-[4] the corresponding choice of classical variable yields a quantum model associated with the choice of constant of the motion as a variable separation constant. This approach works well except for the case of elliptical coordinates [5]. There the constant of the motion associated with variable separation can be taken to be

$$
L=\left(e_{1}-e_{2}\right) A_{1}+e_{2} \mathcal{H}+\left(e_{3}-e_{2}\right) X^{2} .
$$

We can then choose a new basis $L, X, \mathcal{H}$ and $A_{2}$, in which case the relations of the Poisson algebra take the form

$$
\begin{gathered}
\{L, X\}=2\left(e_{1}-e_{2}\right) A_{2},\left\{L, A_{2}\right\}=-2 \frac{\left(e_{1}+e_{2}-2 e_{3}\right)}{\left(e_{1}-e_{2}\right)} L-4 \frac{\left(e_{1}-e_{3}\right)\left(e_{2}-e_{3}\right)}{\left(e_{1}-e_{2}\right)} X^{3} \\
+2 \frac{\left(\left(e_{1}+e_{2}\right) e_{3}-2 e_{1} e_{2}\right)}{\left(e_{2}-e_{1}\right)} X \mathcal{H}+\left(e_{2}-e_{1}\right) \alpha X \\
\left\{A_{2}, X\right\}=\frac{\left(e_{1}+e_{2}\right)}{\left(e_{1}-e_{2}\right)} \mathcal{H}-\frac{2}{\left(e_{1}-e_{2}\right)} L-\frac{\left(e_{1}+e_{2}-2 e_{3}\right)}{\left(e_{1}-e_{2}\right)} X^{2},
\end{gathered}
$$

with corresponding constraint equation

$$
-\frac{1}{\left(e_{1}-e_{2}\right)^{2}} L^{2}+\left[\frac{2 e_{3}-e_{1}-e_{2}}{\left(e_{1}-e_{2}\right)^{2}} X^{2}+\frac{e_{1}+e_{2}}{\left(e_{1}-e_{2}\right)^{2}} \mathcal{H}\right] L+\left[\frac{\left(e_{3}-e_{1}\right)\left(e_{2}-e_{3}\right)}{\left(e_{1}-e_{2}\right)^{2}} X^{4}+\right.
$$




$$
\begin{gathered}
\left.+\frac{\left(2 e_{1} e_{2}-e_{1} e_{3}-e_{2} e_{3}\right)}{\left(e_{1}-e_{2}\right)^{2}} \mathcal{H} X^{2}-\frac{1}{2} \alpha X^{2}\right] \\
-A_{2}^{2}+\frac{1}{4\left(e_{1}-e_{2}\right)^{2}}\left(-2 e_{2} \mathcal{H}+\alpha\left(e_{2}-e_{1}\right)\right)\left(2 e_{1} \mathcal{H}+\alpha\left(e_{2}-e_{1}\right)\right)=0 .
\end{gathered}
$$

Attempting the same procedure as we did with subgroup type coordinates we choose a new set of canonical coordinates $L$ and $\mathcal{H}$ together with their conjugate momenta. If the momentum conjugate to $L$ is $\beta$ then the Poisson bracket relations imply

$$
-\frac{\partial X}{\partial \beta}=2\left(e_{1}-e_{2}\right) A_{2}
$$

Substituting this into the constraint equation we obtain

$$
\begin{gathered}
\left(\frac{\partial X}{\partial \beta}\right)^{2}=-4\left(e_{2}-e_{3}\right)\left(e_{2}-e_{3}\right) X^{4}+4\left[\left(2 e_{3}-e_{1}-e_{2}\right) L+\left(2 e_{1} e_{2}-e_{1} e_{3}-e_{2} e_{3}\right) E-\frac{1}{2} \alpha\right] X^{2} \\
+4\left[-L^{2}+\left(e_{1}+e_{2}\right) E L\right]+\left(2 e_{2} E+\alpha\left(e_{2}-e_{1}\right)\right)\left(-2 e_{2} E+\alpha\left(e_{2}-e_{1}\right)\right) \\
=\Lambda\left(X^{2}-a_{1}\right)\left(X^{2}-a_{2}\right) .
\end{gathered}
$$

The solution can then be expressed in terms of Jacobi elliptic functions as

$$
X=\sqrt{a_{1}} \operatorname{sn}\left(\sqrt{\Lambda a_{2}} \beta, k\right)
$$

where $k^{2}=a_{1} / a_{2}$ and, correspondingly,

$$
A_{2}=\frac{-1}{\left(e_{1}-e_{2}\right)} \sqrt{a_{1} a_{2}} \operatorname{cn}\left(\sqrt{\Lambda a_{2}} \beta, k\right) \operatorname{dn}\left(\sqrt{\Lambda a_{2}} \beta, k\right) .
$$

This does not appear to give any suggestion of a quantum one variable model for $L$ diagonal in terms of differential operators or of difference operators. Indeed we have not been able to quantize this classical model to get directly the spectral resolution of $L$. Instead we adopt a different approach and return to the original superintegrable system to find the eigenvalue equations for the operator $L$. Without loss of generality we can choose $e_{1}=1, e_{2}=0$ and $e_{3}=a=1 / k^{2}$. The Schrödinger equation for the superintegrable system in elliptical coordinates $\rho, \nu$ has the form

$$
\begin{gathered}
\left(-\frac{4}{\rho-\nu}\left[\left[\rho(\rho-1)(\rho-a)\left[\partial_{\rho}^{2}+\frac{1}{2}\left(\frac{1}{\rho}+\frac{1}{\rho-1}+\frac{1}{\rho-a}\right) \partial_{\rho}\right]-\right.\right.\right. \\
\left.\left.\nu(\nu-1)(\nu-a)\left[\partial_{\nu}^{2}+\frac{1}{2}\left(\frac{1}{\nu}+\frac{1}{\nu-1}+\frac{1}{\nu-a}\right) \partial_{\nu}\right]\right]+\frac{\alpha a(a-1)}{(\rho-a)(\nu-a)}-E\right) \Psi=0 .
\end{gathered}
$$


With the identification $a=1 / k^{2}, k^{\prime}=\sqrt{1-k^{2}}$ the separation equations are

$$
\begin{gathered}
\left(\frac{4}{k^{2}} \lambda(\lambda-1)\left(k^{2} \lambda-1\right)\left[\partial_{\lambda}^{2}+\frac{1}{2}\left(\frac{1}{\lambda}+\frac{1}{\lambda-1}+\frac{k^{2}}{k^{2} \lambda-1}\right) \partial_{\lambda}\right]\right. \\
\left.-\alpha \frac{k^{\prime 2}}{k^{2}\left(1-\lambda k^{2}\right)}+E \lambda+\mu\right) \Lambda(\lambda)=0
\end{gathered}
$$

where $\lambda=\rho, \nu$ and $\mu$ is the separation constant. For convenience we make the transformation $\lambda=\left(1-k^{2} \ell\right) / k^{2}$ and write $\Lambda(\lambda) \equiv \mathrm{L}(\ell)$. Then this equation has the form

$$
\begin{gathered}
{\left[\ell(\ell-1)\left(\ell-\frac{1}{k^{\prime 2}}\right)\left[\partial_{\ell}^{2}+\frac{1}{2}\left(\frac{1}{\ell}+\frac{1}{\ell-1}+\frac{1}{\ell-\frac{1}{k^{\prime 2}}}\right) \partial_{\ell}\right]\right.} \\
\left.+\frac{1}{4}\left(\frac{\alpha}{k^{\prime 2} \ell}+E \ell-\frac{1}{k^{\prime 2}}\left(E+k^{2} \mu\right)\right)\right] \mathrm{L}(\ell)=0 .
\end{gathered}
$$

If we now write $\mathrm{L}(\ell)=\ell^{p} L(\ell)$, i.e., make a gauge transformation, then this equation becomes

$$
\begin{gathered}
{\left[\ell(\ell-1)\left(\ell-\frac{1}{k^{\prime 2}}\right)\left[\partial_{\ell}^{2}+\frac{1}{2}\left(\frac{4 p+1}{\ell}+\frac{1}{\ell-1}+\frac{1}{\ell-\frac{1}{k^{\prime 2}}}\right) \partial_{\ell}\right]\right.} \\
\left.+\left[\frac{1}{4}(E+2 p(1+2 p)] \ell-\frac{1}{4 k^{\prime 2}}\left(E+k^{2} \mu\right)-\left(1+\frac{1}{k^{\prime 2}}\right)\right]\right] L(\ell)=0,
\end{gathered}
$$

a form of Heun's equation denoted by the Riemann $P$ symbol [25]

$$
P\left[\begin{array}{cccc}
0 & 1 & 1 / k^{\prime 2} & \infty \\
0 & 0 & 0 & p+r+1 / 4 ; \ell \\
1 / 2-2 p & 1 / 2 & 1 / 2 & p-r+1 / 4
\end{array}\right]
$$

where we have taken $\alpha=2 p(1-2 p)$ and $E=1 / 4-4 r^{2}$. The separation constant $\mu$ appears only in the accessory parameter and not explicitly in the $P$ symbol notation.

Let's now return to the one variable model (9) corresponding to degenerate elliptic coordinates and diagonalize the elliptical coordinate operator $\tilde{L}$ in that model viz $\tilde{L}=A_{1}+X^{2} / k^{2}$. (The eigenvalues of $\tilde{L}$ should just be the values of the separation constant $\mu$ in (11).) If we set $y=\sinh ^{2} t$ then $\tilde{L}$ has the action

$$
\begin{gathered}
\tilde{L} Y(y)=\left[y\left(y-k_{+}\right)\left(y-k_{-}\right)\left[\partial_{y}^{2}+\frac{1}{2}\left(\frac{J+1}{y}+\frac{1-J}{y-k_{+}}+\frac{1-J}{y-k_{-}}\right) \partial_{y}\right]\right. \\
\left.+\frac{1}{8}\left(\alpha+2 J^{2}+E\right)\left(y+\frac{1}{y}\right)+\frac{1}{2} E\right] Y(y)
\end{gathered}
$$


where $k_{+}=\left(1-k^{\prime}\right) /\left(1+k^{\prime}\right)$ and $k_{-}=\left(1+k^{\prime}\right) /\left(1-k^{\prime}\right)$. With the substitution $y=k_{-} z$ this gives $\tilde{L}$ in the form

$$
\begin{aligned}
\tilde{L} \mathrm{Z}(z)=k_{+} & {\left[z\left(z-k_{-}^{2}\right)(z-1)\left[\partial_{z}^{2}+\frac{1}{2}\left(\frac{J+1}{z}+\frac{1-J}{z-k_{-}^{2}}+\frac{1-J}{z-1}\right) \partial_{z}\right]\right.} \\
& \left.+\frac{1}{8}\left(2(E+\alpha)+J^{2}\right)\left(z+\frac{1}{k_{+}^{2} z}\right)+\frac{E}{2 k_{+}}\right] \mathrm{Z}(z) .
\end{aligned}
$$

We look for solutions by writing $\mathrm{Z}(z)=z^{q} Z(z)$. Then for $E=-2(p-q)(2(p-q)+1)$ the operator $\tilde{L}$ assumes the form

$$
\begin{gathered}
\tilde{L} Z(z)=k_{+}\left[z\left(z-k_{-}^{2}\right)(z-1)\left[\partial_{z}^{2}+\frac{1}{2}\left(\frac{J+1+4 q}{z}+\frac{1-J}{z-k_{-}^{2}}+\frac{1-J}{z-1}\right) \partial_{z}\right]\right. \\
\left.+q(1-J) z-q^{2}\left(1+k_{-}^{2}\right)+\frac{E}{2 k_{+}}+\frac{1}{2} k_{-} E\right] Z(z) .
\end{gathered}
$$

Eigenfunctions of this operator are Riemann $P$ functions of the form

$$
P\left[\begin{array}{ccccc}
0 & 1 & k_{-}^{2} & \infty & \\
0 & 0 & 0 & 2 q & ; z \\
(1-J-4 q) / 2 & (J+1) / 2 & (J+1) / 2 & (1-J) / 2
\end{array}\right] .
$$

Again the eigenvalue $\mu$ is incorporated linearly in the accessory parameter, not in the $P$ function notation. We have found two different forms of Riemann $P$ functions, the first by solving the separation equations for the superintegrable system in elliptical coordinates where one separation constant corresponds to $L$, and the second by solving the eigenvalue equation for $L \equiv \tilde{L}$ in the one variable model corresponding to degenerate elliptic coordinates. These two solutions are closely related. Indeed from the identity exhibited in [26], page 59 , viz

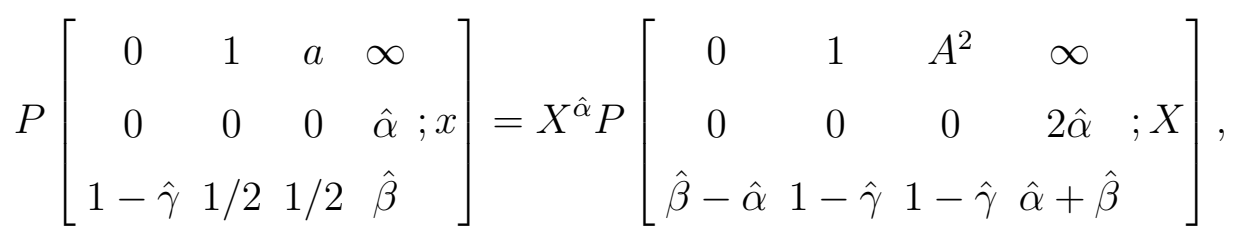

where $A=(1+\sqrt{a}) /(1-\sqrt{a})$ and $X=A(\sqrt{(x-1}+\sqrt{x-a}) /(\sqrt{x-1}-\sqrt{x-a})$. We can now identify the two versions of eigenfunctions of $L$ that we have calculated. By taking $a=1 / k^{\prime 2}$ then $A=\left(k^{\prime}+1\right) /\left(k^{\prime}-1\right)$ and we obtain an identity between the two $P$ function expressions that we have derived, up to a gauge equivalence, by further taking $J=-4 p$, 
$p=q+r+1 / 4$. In this way we have identified the two derived realizations of $L$. Indeed the direct relationship between variables $t$ and $\lambda$ is

$$
\sinh ^{2} t=y=\frac{\left(k^{\prime}+1\right)}{\left(k^{\prime}-1\right)} \frac{(\sqrt{1-\lambda}-i \sqrt{\lambda})}{(\sqrt{1-\lambda}+i \sqrt{\lambda})} .
$$

This example has illustrated the relationship between the two different ways of realizing the operator $L$. It also appears that

the classical model in elliptical coordinates does not suggest a suitable quantum mechanical model, but this may just be due to our lack of insight thus far.

\section{DISCUSSION AND OUTLOOK}

As shown in Reference 11, up to equivalence under the Stäckel transform between manifolds, there are exactly 13 second order 2D superintegrable systems, 6 corresponding to degenerate (1-parameter) potentials and 7 to nondegenerate (3-parameter) potentials. In each case the second order symmetries generate a finite dimensional algebra polynomially closed under commutation, the quadratic algebra. These algebras and their representation theory are reminiscent of semi-simple Lie algebras, but definitely distinct. Realizations of the representations in terms of differential and difference operator models have independent interest, partly due to deep connections with the theory of orthogonal polynomials. For degenerate potentials the algebra of first and second order symmetries closes at order 4 and the structure is especially simple. In this paper we have chosen one such case and described how models of the classical superintegrable system can be determined in a straightforward manner, associated one-to-one with the possible orthogonal coordinates in which the associated Hamilton-Jacobi equation permits additive separation. Each model essentially gives the spectral resolution of the constant of the motion associated with the variable separation. To get one variable models of the quantum quadratic algebra from the classical models we have to solve the quantization problem. In our example we showed that this is easy to do, except in the case of generic ellipsoidal coordinates where no differential or difference operator model exists. However, one can still determine the spectral resolution of the operator corresponding to elliptical coordinates by computing in one of the other models. In this case we show that the eigenvalue equation in a certain one variable model corresponds exactly to one of the separation equations for solving the original Schrödinger equation in elliptical 
coordinates.

For 2D systems with nondegenerate potential the quadratic algebra closes at level 6 and the representation theory is more complicated. However, the same basic features demonstrated in our example still hold. Indeed, this example is typical of 2D superintegrable systems in general, as will be shown in the thesis [27] of S. Post. The connection with orthogonal polynomials is striking for nondegenerate potentials. For example, the quadratic algebra of the generic superintegrable system on the two sphere is exactly the one that describes the Wilson and Racah polynomials in their full generality! Thus Wilson and Racah polynomials are deducible directly from classical mechanics.

For 3D superintegrable systems the structure is much more complicated. For nondegenerate (4-parameter potentials) where $2 n-1=5$ there are 5 functionally independent second order symmetries, but always 6 linearly independent symmetries. The 6 symmetries generate a quadratic algebra that always closes at order 6 , Further the functional dependence of the 6 linearly independent symmetries is given by an identity of order 8 in the momentum variables. We have begun the study of the representation theory and two variable models for representations of the quantum quadratic algebras of these systems, initially focusing on the 3D singular isotropic oscillator. We have already shown that models for this system correspond to differential recurrence relations for Jacobi polynomials in one case, to differential-difference equations for families of dual Hahn polynomials in another and to difference-difference operator equations relating distinct families of Wilson polynomials in a third. The generic superintegrable system on the three sphere will correspond to two variable extensions of Wilson polynomials. This work is in progress,

[1] E. G. Kalnins, W. Miller, Jr., G. C. Williams and G. S. Pogosyan, J. Phys. A: Math Gen. 35, 4655 (2002).

[2] N. W. Evans, Phys. Rev. A. 41, 5666 (1990).

[3] M. F. Rañada, J. Math. Phys. 38, 4165 (1997).

[4] Superintegrability in Classical and Quantum Systems, P. Tempesta, P. Winternitz, W. Miller, G. Pogosyan editors, AMS, 37, 2005.

[5] G. Koenigs. Sur les géodésiques a intégrales quadratiques. A note appearing in "Lecons sur la 
théorie générale des surfaces". G. Darboux. Vol 4, 368-404, Chelsea Publishing 1972.

[6] E. G. Kalnins, J. M. Kress and W. Miller, Jr., J. Math. Phys. 46, 053509 (2005).

[7] E. G. Kalnins, J. M. Kress and W. Miller, Jr., J. Math. Phys. 47, 043514 (2006).

[8] C. Daskaloyannis and Y. Tanoudis, J. Math Phys. 48, 072108 (2007)

[9] E. G. Kalnins, J. M. Kress and P. Winternitz, J. Math. Phys. 43, 970 (2002).

[10] E. G. Kalnins, J. M. Kress, W. Miller, Jr. and P. Winternitz, J. Math. Phys. 44, 5811 (2003).

[11] J. M. Kress, Phys. Atomic Nuclei 70, 560 (2007).

[12] E. G. Kalnins, W, Miller, Jr. and S. Post, J. Phys. A: Math. Theor. 40, 11525 (2007).

[13] E. G. Kalnins, W, Miller, Jr. and S. Post, SIGMA 4, 008 arXiv:0801.2848 (2008).

[14] E. G. Kalnins, J. M. Kress, W. Miller, Jr., and G. S. Pogosyan, J. Phys. A: Math Gen. 34, 4705 (2001).

[15] E. G. Kalnins, J. M. Kress and W. Miller, Jr., J. Math. Phys. 47, 093501 (2006).

[16] D. Bonatos, C. Daskaloyannis and K. Kokkotas, Phys. Rev. A. 50, 3700 (1994).

[17] C. Daskaloyannis, J. Math. Phys. 42, 1100 (2001).

[18] Y. I. Granovskii, A. S. Zhedanov and I. M. Lutsenko, Theoret. and Math. Phys. 91, 604 (1992).

[19] C. Daskaloyannis C., J. Math. Phys 42, 1100 math-ph/0003017 (2001).

[20] C. Quesne, Phys. Lett. A 193, 245 (1994).

[21] C. Daskaloyannis and K. Ypsilantis, J. Math Phys. 47, 042904 (2006).

[22] C. Quesne, SIGMA 3, 067 (2007).

[23] G. E. Andrews, R. Askey and R. Roy, Special Functions. Encyclopedia of Mathematics and its Applications, Cambridge University Press, Cambridge, UK, 1999.

[24] E. G. Kalnins, Separation of Variables for Riemannian Spaces of Constant Curvature, Pitman, Monographs and Surveys in Pure and Applied Mathematics 28, Longman, Essex, England, 1986.

[25] E. G. C. Poole, Introduction to the Theory of Linear Differential Equations, Dover, New York, 1960.

[26] A. Erdélyi, W. Magnus, F. Oberhettinger, and F. G. Tricomi, Higher Transcendental Functions, Volume III, McGraw-Hill, New York, 1955.

[27] S. Post, University of Minnesota Ph.D. Thesis, Second order superintegrable systems, (in preparation), 2009. 